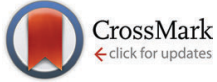

Cite this: Phys. Chem. Chem. Phys., $2015,17,8670$

Received 15th December 2014, Accepted 19th February 2015

DOI: $10.1039 / c 4 c p 05865 h$

www.rsc.org/pccp

\section{Electrochemical in situ investigations of SEI and dendrite formation on the lithium metal anode}

\begin{abstract}
Georg Bieker, Martin Winter and Peter Bieker*
This comparative work studies the self-enforcing heterogeneity of lithium deposition and dissolution as the cause for dendrite formation on the lithium metal anode in various liquid organic solvent based electrolytes. In addition, the ongoing lithium corrosion, its rate and thus the passivating quality of the SEI are investigated in self-discharge measurements. The behavior of the lithium anode is characterized in two carbonate-based standard electrolytes, $1 \mathrm{M} \mathrm{LiPF}_{6}$ in EC/DEC (3:7) and $1 \mathrm{M} \mathrm{LiPF} 6$ in EC/DMC (1:1), and in two alternative electrolytes $1 \mathrm{M} \mathrm{LiPF}_{6}$ in TEGDME and $1 \mathrm{M}$ LiTFSI in DMSO, which have been proposed in the literature as promising electrolytes for lithium metal batteries, more specifically for lithium/air batteries. As a result, electrolyte decomposition, SEI and dendrite formation at the lithium electrode as well as their mutual influences are understood in the development of overpotentials, surface resistances and lithium electrode surface morphologies in subsequent lithium deposition and dissolution processes. A general model of different stages of these processes could be elaborated.
\end{abstract}

\section{Introduction}

The lithium metal anode provides a very high capacity and the lowest potential of all metallic anode materials. ${ }^{1-3}$ Therefore, it is not only used in commercial primary lithium metal batteries, but is also proposed as an anode material in rechargeable lithium/air ${ }^{4,5}$ and lithium/sulfur batteries, which are considered as super-high specific energy accumulators of tomorrow. These high energy batteries are urgently demanded to meet a longer driving range in electric vehicles (electro-mobility). ${ }^{6}$

However, the rechargeable lithium metal anode suffers from poor rechargeability and low safety. ${ }^{2,3,7}$ Due to the low potential the electrolytes used are thermodynamically not stable against lithium. Their reductive decomposition and the parallel corrosion of the Li electrode lead to the formation of the solid electrolyte interphase (SEI). ${ }^{8,9}$ This passivating film is supposed to slow down or in the ideal case even prevent electrolyte decomposition. ${ }^{1,2,10-14}$ In addition, heterogeneous lithium deposition and dissolution during charge and discharge of the lithium metal anode eventually leads to high surface area lithium, commonly called lithium dendrites in most of the organic solvent-based electrolytes..$^{2,3,7,15-18}$ This may cause a loss of active material due to enhanced lithium corrosion at the high surface area $\mathrm{Li}$, as well as due to the disconnection of dendrites from electronic contact. In addition, short-circuit of the cell may happen when the dendrites grow across the

MEET Battery Research Center, Institute of Physical Chemistry, University of Münster, Corrensstrasse 28/30, 48149 Münster, Germany. E-mail: peter.bieker@uni-muenster.de electrolyte to the cathode. In any case, the continuous creation of new lithium surfaces by dendrite formation leads to continuous electrolyte decomposition during cycling.

For a better understanding of these phenomena and their mutual influences it appears favourable to take a closer look at the kinetics of lithium dissolution (= discharge, stripping) and deposition (= charge, plating) of/at the lithium metal anode. As these are represented by overpotentials, we developed a method to observe dendrite formation in situ in constant current cycling experiments. To exclude effects from a nonlithium metal cathode material, these experiments were carried out in symmetrical $\mathrm{Li} / \mathrm{Li}$ cells. Electrochemical impedance spectroscopy (EIS) and scanning electron microscopy (SEM) were used to support the interpretation of the overpotentials by measuring the development of the electrode surface resistance (EIS) and its morphology (SEM) during subsequent lithium deposition and dissolution processes.

The second part of this study concentrates on the investigation of SEI formation and the passivating properties of the SEI by deposition and dissolution of lithium on/of a $\mathrm{Cu}$ foil as a counter electrode (CE). The difference between the charge invested for the deposition process and the charge gained for lithium dissolution is a rough but fast indicator for the amount of the deposited lithium that has been corroded or has lost electronic contact. Additional information about the protective properties of the SEI was gathered by detecting the rate of the ongoing lithium corrosion in self-discharge measurements. In these experiments, the difference in Coulombic efficiency during lithium deposition and dissolution on $\mathrm{Cu}$ depends on a rest time interval between the deposition and dissolution processes. 
The above experiments were carried out as comparative investigations in various liquid electrolytes. Therefore, the performance of the lithium metal anode in $1 \mathrm{M}$ lithium hexafluorophosphate $\left(\mathrm{LiPF}_{6}\right)$ in ethylene carbonate (EC)/diethyl carbonate (DEC) $(3: 7),{ }^{5,16,19-25} 1 \mathrm{M} \mathrm{LiPF}_{6}$ in ethylene carbonate (EC)/dimethyl carbonate (DMC) $(1: 1),,^{5,18,20,22-25} 1 \mathrm{M} \mathrm{LiPF}_{6}$ in tetraethylene glycol dimethyl ether (TEGDME) $)^{4,26-33}$ and $1 \mathrm{M}$ lithium bis(trifluoromethanesulfonyl)imide (LiTFSI) in dimethyl sulfoxide (DMSO) ${ }^{4,29,34}$ was investigated. As EC/DEC- and EC/ DMC-based electrolytes are the broadly investigated state-of-theart electrolytes in lithium-ion batteries, ${ }^{35,36}$ they were chosen as a standard system. The TEGDME- and DMSO-based electrolytes were chosen because they have been reported as promising electrolytes for lithium/air batteries. The investigations of the lithium anode surface in TEGDME-based electrolytes might be of additional special importance as many polymer-based electrolytes use polyethers, such as polyethylene oxide (PEO) and poly[bis((methoxyethoxy)ethoxy)phosphazene] (MEEP) with similar functional groups. ${ }^{37}$

\section{Results and discussion}

The potential of the lithium metal working electrode (WE) during constant current lithium deposition and dissolution in a $\mathrm{Li} / \mathrm{Li}$ cell containing $1 \mathrm{M} \mathrm{LiPF}_{6}$ in EC/DEC (3:7) electrolyte is presented in Fig. 1. The positive potentials of the Li WE against the $\mathrm{Li} / \mathrm{Li}^{+}$reference electrode ( $\mathrm{RE}$ ) represent the overpotentials appearing during lithium dissolution, whereas the negative potentials represent the overpotentials during lithium deposition on the WE. This experiment shows that the overpotentials of both the lithium deposition and dissolution processes strongly decrease under repeated cycling.

Overpotentials are generated by kinetic hindrances in the system. In lithium plating and stripping processes, these may include the lithium ion transport in the electrolyte and in the electrode/electrolyte interphase, such as the SEI, and always the kinetic hindrance of the lithium ion reduction and oxidation processes at the electrode itself, influencing the charge transfer resistance. The contributions of these processes to the overall cell resistance can be identified by electrochemical impedance spectroscopy (EIS).

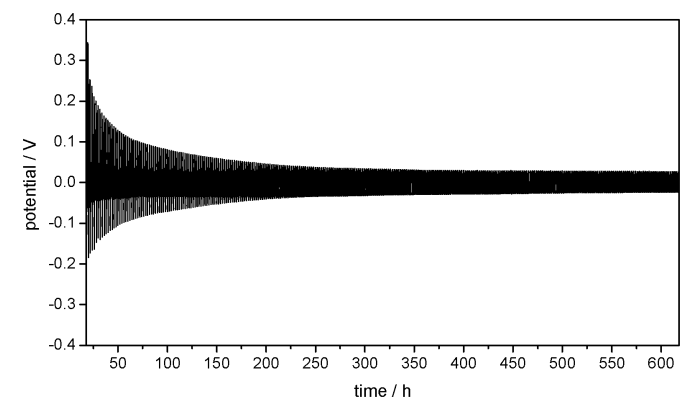

Fig. 1 Development of overpotentials during subsequent lithium plating/ stripping processes on the WE in Li/Li symmetrical cells with a Li reference electrode containing $1 \mathrm{M} \mathrm{LiPF}_{6}$ in $\mathrm{EC} \mathrm{DEC}(3: 7)$ as the electrolyte at $j=$ $0.1 \mathrm{~mA} \mathrm{~cm}^{-2}$.
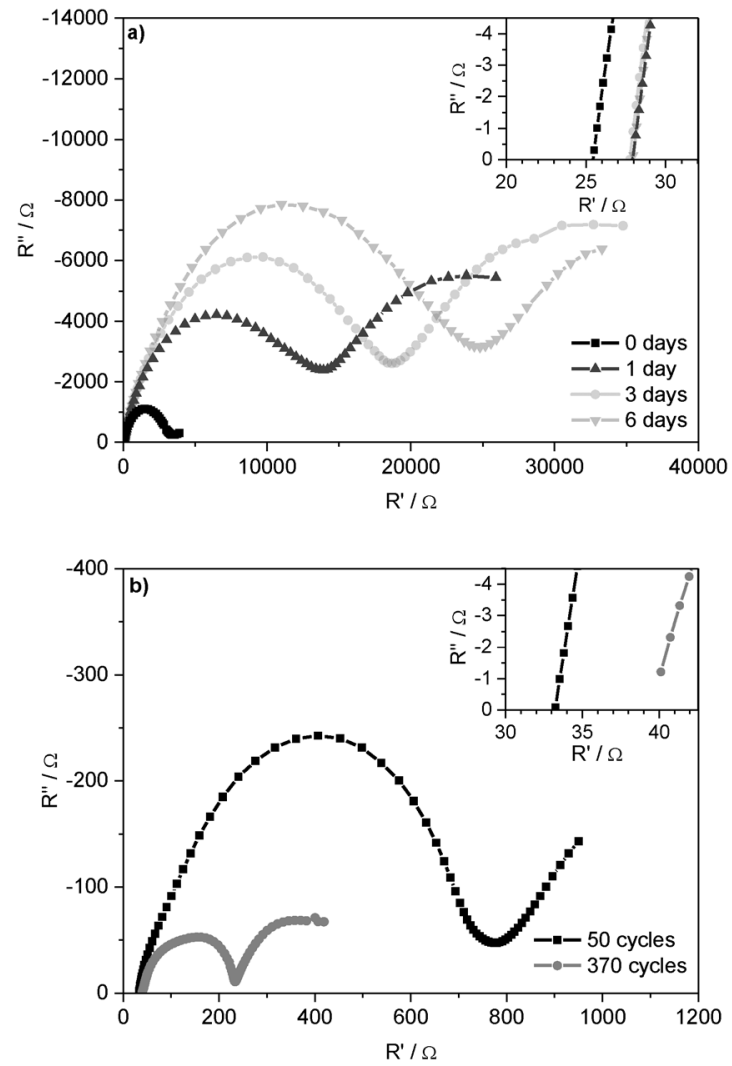

Fig. 2 Nyquist plot of the impedance spectra of Li/Li cells with $1 \mathrm{M} \mathrm{LiPF}_{6}$ in EC/DEC (3:7) as the electrolyte (a) after $0,1,3$ and 6 days under OCP conditions and (b) after 50 and 370 lithium plating/stripping cycles at $j=$ $0.1 \mathrm{~mA} \mathrm{~cm}^{-2}$.

Fig. 2a presents the Nyquist plot of impedance spectra of symmetrical $\mathrm{Li} / \mathrm{Li}$ cells after $0,1,3$, and 6 days under open circuit potential (OCP) conditions. The spectra start with the Ohmic resistance and then show a semicircle at lower frequencies. Whereas the Ohmic part of the cell resistance is determined by the ionic conductivity of the electrolyte ( $\left.R_{\text {electrolyte }}\right)$, the following semicircle consequently corresponds to the processes at/on the two electrodes: capacitive properties of the grain boundary resistances in the SEI and the charge-transfer resistances at the Li electrodes. ${ }^{9,38}$ As these resistances occur on/at the surface of the Li electrodes (including the SEI), they are summarized (see Experimental part) and interpreted as ' $\mathrm{Li}$ electrode surface resistance' $\left(R_{\text {surface}}\right)$. The determined resistance values are given in Table 1 . Based on the native surface film on the untreated $\mathrm{Li}$ foil, this increase indicates further

Table 1 Electrolyte resistance $R_{\text {electrolyte }}$ and surface resistance $R_{\text {surface }}$ of $\mathrm{Li} / \mathrm{Li}$ cells with $1 \mathrm{M} \mathrm{LiPF}_{6}$ in EC/DEC $(3: 7)$ as the electrolyte before and after 6 days under OCP conditions and after 50 and 370 lithium plating/stripping cycles at $j=0.1 \mathrm{~mA} \mathrm{~cm}^{-2}$

\begin{tabular}{llr}
\hline Cycling conditions & $R_{\text {electrolyte }} / \Omega$ & $R_{\text {surface }} / \Omega$ \\
\hline Uncycled, 0 d & 26 & 3255 \\
Uncycled, 6 d & 28 & 25089 \\
50 cycles, 5 d & 33 & 760 \\
370 cycles, 32 d & 39 & 237
\end{tabular}


formation of the SEI with its bulk and grain boundary resistances. Under OCP conditions, these resistances converge to the maximal values after several days. Hence, the SEI thickness and composition are deduced to remain constant.

These experiments also revealed that the Ohmic resistance of the electrolyte $R_{\text {electrolyte }}$ is far lower than the resistances at the electrode surface $R_{\text {surface }}$. The same could be observed for the other electrolytes. Therefore, it can be assumed that the overpotential of lithium deposition and dissolution processes is determined by the nature of the SEI and the processes at the lithium/electrolyte interface.

Fig. 2b shows the Nyquist plot of the impedance spectra after 50 and 370 subsequent lithium deposition and dissolution processes, i.e. after 50 and 370 'cycles'. The comparison of the resistances in the cells under OCP conditions with the resistances of the cycled cells is given in Table 1 .

It can be seen that the surface resistance $R_{\text {surface }}$ of the cells after 50 lithium plating/stripping cycles is one order of magnitude lower than the surface resistance of the cell measured directly after assembly ( 0 days) and two orders of magnitude lower than that measured after 6 days under OCP conditions. This effect correlates with the strong decrease of the overpotentials in the plating/stripping experiments (compare Fig. 1) and can be explained by a large increase in the surface area of the lithium anode during cycling. The different shapes of the impedance spectra are coherent with the changing lithium electrode surface morphology and the chemically different SEI after 50 and 370 cycles.

Besides the general decrease of the overpotentials the shape of the overpotential profiles also changes during cycling (Fig. 3a). Long-term cycling experiments show that the overpotential profiles become constant after a certain number of cycles. As the overpotential profiles change continuously from the first cycle to the cycle where the profile shape gets constant these two potential profiles will be discussed in detail.

The first lithium deposition process starts with an immediate drop in the potential (to $-0.3 \mathrm{~V}$ at $j=0.1 \mathrm{~mA} \mathrm{~cm}^{-2}$, Fig. 3a) (region A). This large drop, which is the maximum overpotential in the whole experiment, can be explained by a highly specific kinetic hindrance for lithium deposition underneath the initial electrode surface film. It is induced by a smooth Li metal surface with a resistive interphase that consists of a native surface film of the untreated lithium metal electrode (the 'Li substrate') and the SEI formed spontaneously after immersion of lithium in the electrolyte. The related surface resistance $R_{\text {surface }}$ is observed in the EIS measurements.

Only after very little current flow, the overpotential is cut down immediately to around $-0.1 \mathrm{~V} v s . \mathrm{Li} / \mathrm{Li}^{+}$(at $j=$ $0.1 \mathrm{~mA} \mathrm{~cm}^{-2}$ ), continues to decrease rapidly at first and then decreases slower and slower. This decrease of the overpotential during lithium deposition (region B) can be explained by deposition of new lithium on previously deposited lithium. The chemical composition of the SEI as well as a nonuniform morphology of the lithium electrode (e.g. sharp edges, cracks, and holes in nanometer to micrometer scale $)^{18}$ lead to a nonuniform current distribution when an electric field is

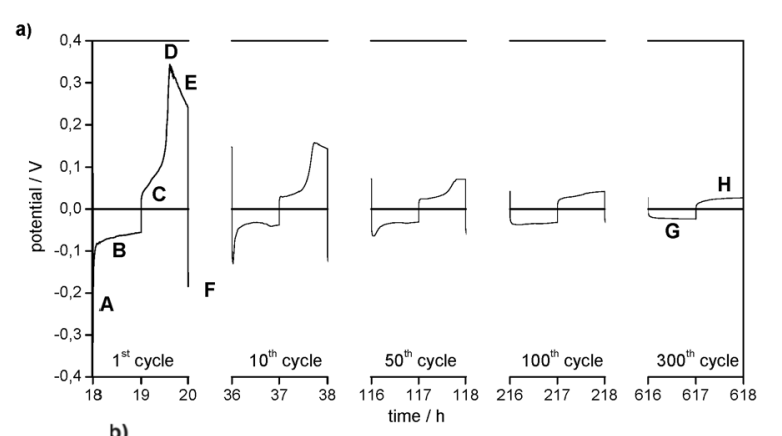

b) $\mathrm{A}$
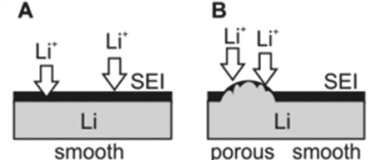

D

E
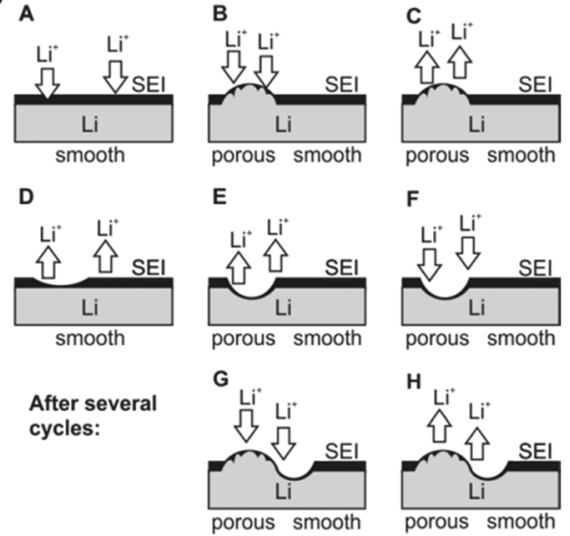

Fig. 3 (a) Selected overpotential profiles of subsequent lithium plating/ stripping processes on the WE, denoted as 'cycles', in Li/Li symmetrical cells with $1 \mathrm{M} \mathrm{LiPF}_{6}$ in EC/DEC $(3: 7)$ as the electrolyte at $j=0.1 \mathrm{~mA} \mathrm{~cm}^{-2}$. (b) Heterogeneous lithium deposition $(A+B+F+G)$ and dissolution $(C+D+E+H)$ on/at the lithium metal anode. Please note that the pristine $\mathrm{SEI}$ is thick and resistive, whereas the 'fresh' SEI on the 'fresh' lithium deposit is thin and not that resistive. The resistances are not only influenced by the different thicknesses of the SEI, but also by their chemical compositions.

applied and thus locally preferred lithium deposition (and dissolution). As the surface film has limited flexibility, it can only accommodate a certain volume change due to lithium deposition (or dissolution) and breaks. After cracking the initial surface layer (native surface film plus largely formed SEI) the protruding tips of 'fresh' deposited lithium offer a stronger electrical field, a higher specific surface area and thus a higher interface area with the electrolyte and lower bulk and grain boundary resistances due to the broken native surface film and an incompletely formed and thus also chemically different surface film. Consequently, further lithium deposition concentrates on previously deposited lithium. The self-amplifying effect of heterogeneous lithium deposition is observed.

In the later cycles the negative overpotentials in region $\mathrm{A}$ are reduced to one order of magnitude lower values. This indicates a general decrease of the overall resistance $v s$. lithium deposition. The corresponding decrease of the surface resistances $R_{\text {surface }}$ is detected in EIS measurements (see Fig. 2 and Tables 1 and 2). It can be concluded that, whereas in the first cycles almost the whole Li electrode surface is smooth and covered by the initial surface film and a further formed SEI, the roughened parts of the electrode surface are observed where heterogeneous lithium deposition/dissolution took place before their 
Table 2 Electrolyte resistance $R_{\text {electrolyte }}$ and surface resistance $R_{\text {surface }}$ of $\mathrm{Li} / \mathrm{Li}$ cells with $1 \mathrm{M} \mathrm{LiPF}_{6}$ in EC/DEC (3:7) (EC/DEC), $1 \mathrm{M} \mathrm{LiPF}{ }_{6}$ in EC/DMC (1:1) (EC/DMC), 1 M LiPF 6 in TEGDME (TEGDME), and $1 \mathrm{M}$ LiTFSI in DMSO (DMSO) as electrolytes before and after $x$ days under OCP conditions and after 50 and $y$ lithium plating/stripping cycles at $j=0.1 \mathrm{~mA} \mathrm{~cm}^{-2}(x=6$ for EC/DEC, 4 for EC/DMC, 2 for TEGDME, 7 for DMSO; $y=370$ for EC/DEC and EC/DMC, 230 for TEGDME, 210 for DMSO)

\begin{tabular}{lrrrr}
\hline & EC/DEC & EC/DMC & TEGDME & DMSO \\
\hline$R_{\text {electrolyte }}($ OCP, 0 days) $/ \Omega$ & 26 & 25 & 93 & 436 \\
$R_{\text {electrolyte }}($ OCP,$x$ days $) / \Omega$ & 28 & 18 & 101 & 344 \\
$R_{\text {electrolyte }}(50$ cycles $) / \Omega$ & 33 & 31 & 195 & 235 \\
$R_{\text {electrolyte }}(y$ cycles $) / \Omega$ & 39 & 34 & 744 & 151 \\
$R_{\text {surface }}($ OCP, 0 days $) / \Omega$ & 3255 & 3472 & 2217 & 1619 \\
$R_{\text {surface }}($ OCP,$x$ days $) / \Omega$ & 25089 & 10203 & 9173 & 4402 \\
$R_{\text {surface }}(50$ cycles $) / \Omega$ & 760 & 765 & 450 & 2682 \\
$R_{\text {surface }}(y$ cycles $) / \Omega$ & 237 & 207 & 59698 & 3415 \\
\hline
\end{tabular}

growth. As a result, the overall surface resistance $R_{\text {surface }}$ and thus the initial overpotential of lithium deposition decrease. When the potential profile shape becomes constant, e.g. in cycle 300 , the negative potential starts with a small drop and then increases slowly. Similar to region B this behaviour is assumed to refer to lithium deposition on parts of the Li electrode surface where not only subsequent heterogeneous lithium deposition but also dissolution has roughened the surface and changed the surface film (region G).

The overpotential region of the first lithium dissolution processes in Fig. 3a is divided into three parts. In the first part, it increases slightly (C) and in the second part it increases steeply until it reaches a maximum (D), whereas the third part shows a decrease of the overpotential (E). However, when the experiment starts with a lithium dissolution process as in the plating/stripping experiments with $\mathrm{Li} / \mathrm{Cu}$ cells (compare Fig. 3 with the 1st cycle of Fig. 4), only the electrode behaviour indicated by the regions $\mathrm{D}$ and $\mathrm{E}$ is observed. The experiments with changing charge capacities of the Li WE (compare with Fig. 11a and b) indicate that the capacity of the preceding lithium deposition process correlates with the duration of the first region (C) of the following lithium dissolution process.

It can be concluded that region $\mathrm{C}$ of the dissolution overpotential profile is determined by the dissolution of 'fresh' deposited or roughened lithium, i.e. by Li with a high surface area and a not well-developed thin SEI. This at least partial

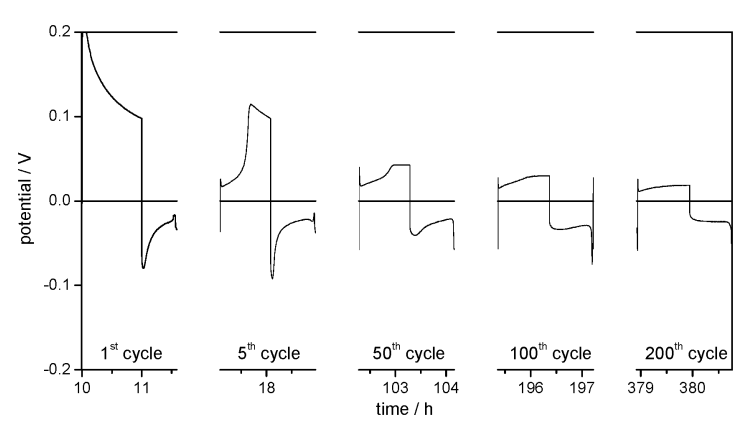

Fig. 4 Selected overpotential profiles of subsequent lithium plating/stripping processes on the WE, denoted as 'cycles', in $\mathrm{Li}$ (WE)/Cu (CE) cells with $1 \mathrm{M} \mathrm{LiPF}_{6}$ in EC/DEC $(3: 7)$ as the electrolyte at $j=0.1 \mathrm{~mA} \mathrm{~cm}^{-2}$. re-dissolution of dendritic lithium was observed in previous publications ${ }^{18}$ and was regarded as a cause for the loss of contact of dendrites from the substrate and thus the formation of electrically isolated lithium. ${ }^{10,39,40}$

As the 'fresh' deposited or roughened lithium is still limited in amount in the first cycles, the rapid increase of the overpotential indicates the end of the corresponding dissolution process. Consequently, the high overpotentials (region D) are merely allocated to the dissolution of 'old' lithium underneath the initial surface film and the dissolution of the Li substrate which was previously covered by 'fresh' deposited lithium. During the first cycles the overpotentials of lithium dissolution in region $\mathrm{D}$ are high because the process primarily takes place on the pristine, thus smooth and low surface area Li substrate. The decrease of the overpotential in region $\mathrm{D}$ after several cycles confirms that the corresponding lithium dissolution increasingly takes place from an already roughened lithium surface.

Analogous to dendrite formation during lithium deposition lithium dissolution also changes the electrode surface morphology. ${ }^{18}$ The nonuniform current distribution leads to the formation of holes, which can even cause the breaking of the SEI. The corresponding increase in the electrode surface area and a simultaneous decrease of the surface layer resistance lead to a locally preferred, thus inhomogeneous lithium dissolution. As for $\mathrm{Li}$ deposition in region $\mathrm{A}$, the decrease of the overpotential in region $\mathrm{E}$ indicates this process during Li dissolution. The already rough parts of the Li electrode surface are roughened further.

As the local roughness also makes these spots preferable for lithium deposition, it can be deduced that these areas grow during repeated lithium deposition and dissolution cycles. As a result, lithium dissolution increasingly takes place on the porous areas and thus the overpotentials in region $\mathrm{D}$ are declining continuously. Finally, when the overpotential profiles during lithium dissolution become constant, e.g. in cycle 300, region D completely disappears from the profile. As for region C, it can be concluded that from this time onward lithium dissolution predominantly takes place on the porous areas of the lithium electrode (region G).

In order to verify the conclusions of this model, scanning electron micrographs (SEM) were taken from Li electrodes before/after 50 and 370 lithium plating/stripping cycles at $j=$ $0.1 \mathrm{~mA} \mathrm{~cm}^{-2}$ in symmetrical Li/Li cells. Fig. 5 shows the Li WE surface after 370 cycles. The electrode surface is divided into locally bordered smooth and rough or rather dendritic areas. SEM analyses of the uncycled Li electrodes determine that the rough areas appear only after lithium plating/stripping cycles and the smooth areas correspond to the surface of the still uncycled Li. The local concentration and the clear borders of the rough areas confirm the conclusion of the strong preference of lithium dissolution and deposition processes on areas where these processes already took place.

For a more general understanding of the lithium deposition and dissolution behaviour on/of the Li metal anode in liquid electrolytes, these basic investigations were expanded to three 


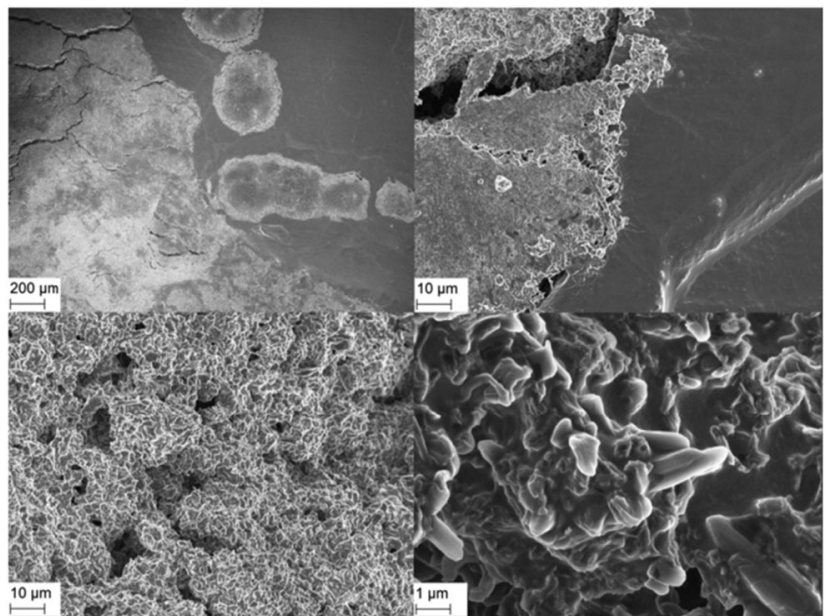

Fig. 5 SEM images (EHT $=3.00 \mathrm{kV}$ ) of the Li WE (unwashed) after 370 cycles at $j=0.1 \mathrm{~mA} \mathrm{~cm}{ }^{-2}$ in $1 \mathrm{M} \mathrm{LiPF}_{6}$ in EC/DEC (3:7) at $50 \times$ (top left), $1 \mathrm{k} \times$ (top right, bottom left) and $10 \mathrm{k} \times$ (bottom right) magnification.

further electrolytes: $1 \mathrm{M} \mathrm{LiPF}_{6}$ in EC/DMC (1:1), $1 \mathrm{M} \mathrm{LiPF}_{6}$ in TEGDME, and $1 \mathrm{M}$ LiTFSI in DMSO. During lithium plating/ stripping experiments in $\mathrm{Li} / \mathrm{Li}$ symmetrical cells several similarities between the electrolytes can be observed. Accordingly, the interpretation of the overpotential profiles in the EC/DECbased electrolyte (Fig. 3) can be used as a reference. In comparison to this reference, the individual deviations of the other electrolytes are discussed in the following.

Fig. 6 shows the overpotential profiles of the lithium deposition and dissolution processes in $1 \mathrm{M} \mathrm{LiPF}_{6}$ in an EC/DMC (1:1) electrolyte. These profiles, the development of the electrode surface resistances in EIS (Table 2) and the morphology of the Li electrodes before and after the plating/stripping cycles are analogous to the EC/DEC-based reference electrolyte and thus confirm the model.

For the Li WE in $1 \mathrm{M} \mathrm{LiPF}_{6}$ in TEGDME electrolyte (Fig. 7), the overpotential profiles and the general decrease of the potentials in the early cycles confirm the model as well. Different from the EC/DEC- and EC/DMC-based electrolytes, the SEM investigations show the formation of a surface film after only 50 cycles. However, under this film, the SEM images show locally separated rough and smooth areas on the $\mathrm{Li}$

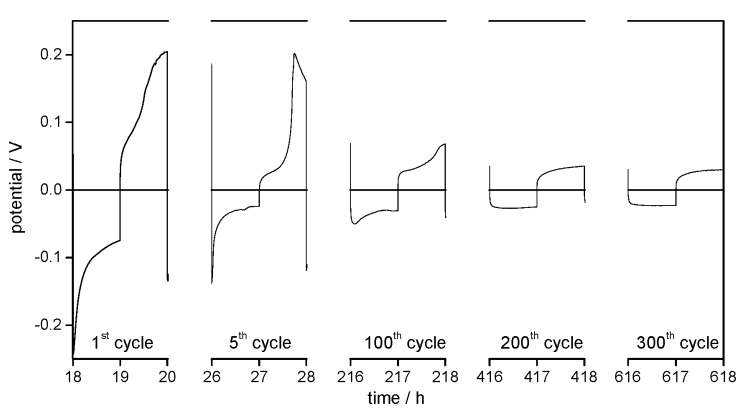

Fig. 6 Selected overpotential profiles of subsequent lithium plating/stripping processes on the WE in Li/Li cells with $1 \mathrm{MLiPF}_{6}$ in EC/DMC $(1: 1)$ as the electrolyte at $j=0.1 \mathrm{~mA} \mathrm{~cm}^{-2}$.
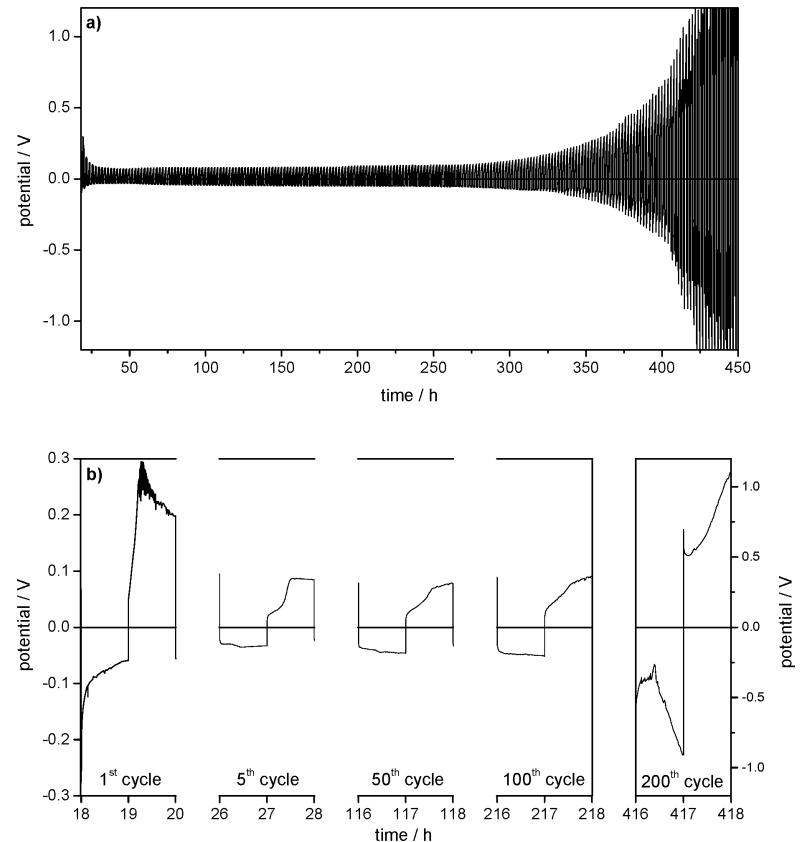

Fig. 7 (a) Overview of the development of overpotentials during cycling and (b) selected overpotential profiles of subsequent lithium plating/ stripping processes on the WE in Li/Li symmetrical cells with $1 \mathrm{M} \mathrm{LiPF}_{6}$ in TEGDME as the electrolyte at $j=0.1 \mathrm{~mA} \mathrm{~cm}^{-2}$.

electrode, and the EIS investigations (Table 2) also detect a decrease of the surface resistances $R_{\text {surface }}$ after 50 cycles.

So far, the processes on the $\mathrm{Li}$ electrode have shown the same behaviour as in the two investigated carbonate based electrolytes. However, after several hundred cycles the overpotentials of lithium deposition and dissolution in the TEGDME-based electrolyte increase exponentially until the experiment has to be stopped. This behaviour was found to be highly reproducible upon several repetitions of the experiment. It can be concluded that the strong increase of the cell resistance is caused by a large degradation of the electrolyte on the Li electrode.

The EIS measurements (Table 2) confirm an increase of the electrolyte resistance $R_{\text {electrolyte }}(195$ to $744 \Omega$ ) between the 50th and 230th cycle, and also show a dramatic increase of the surface resistance $R_{\text {surface }}(450$ to $59698 \Omega$ ). It can be deduced that the increase of the overpotentials is primarily due to the formation of an insulating surface film, as the SEM investigations show a large growth of the surface layer between the 50th and 230th cycle.

The lithium plating/stripping experiments in the $1 \mathrm{M}$ LiTFSI in DMSO electrolyte at $j=0.1 \mathrm{~mA} \mathrm{~cm}{ }^{-2}$ reproducibly show a high fluctuation and noise of the absolute potential values (see forthcoming Fig. 9b) and the shape of the overpotential profiles. In addition, high resistances at the electrode surface $R_{\text {surface }}$ after 50 and 210 cycles are observed in EIS measurements (Table 2). The EIS investigations reveal the formation of a resistive surface film. Both observations can be explained by severe lithium corrosion/electrolyte decomposition during lithium deposition. 


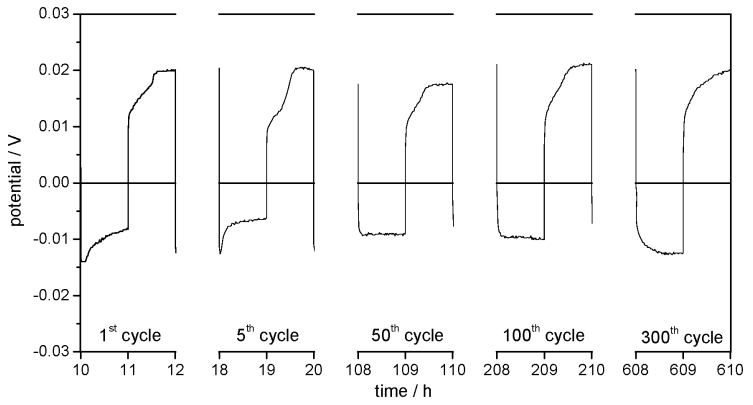

Fig. 8 Selected overpotential profiles of subsequent lithium plating/stripping processes on the WE in Li/Li symmetrical cells with $1 \mathrm{M}$ LiTFSI in DMSO as the electrolyte at $j=0.01 \mathrm{~mA} \mathrm{~cm}^{-2}$.

The lithium deposition and dissolution processes at the one order of magnitude lower current density of $j=0.01 \mathrm{~mA} \mathrm{~cm} \mathrm{~cm}^{-2}$ (Fig. 8) show low overpotentials of -10 up to $-15 \mathrm{mV}$ for the lithium deposition and between 18 and $22 \mathrm{mV}$ for the lithium dissolution over 300 cycles, which stay almost constant during the whole experiment. Compared to the experiment at $j=$ $0.1 \mathrm{~mA} \mathrm{~cm} \mathrm{~cm}^{-2}$, the overpotentials are reduced by more than a factor of 10 (compare Fig. 9a and b).

Apparently, the surface resistance $R_{\text {surface }}$ has increased less and thus less electrolyte degradation takes place. This may be also explained by a lower amount of dendrites, which are more reactive with the electrolyte. Generally, it can be stated that the extent of degradation of DMSO and/or the LiTFSI salt on the Li electrode strongly depends on the current density.

The shape of the overpotential profiles in Fig. 8 shows the same development during cycling as discussed for the
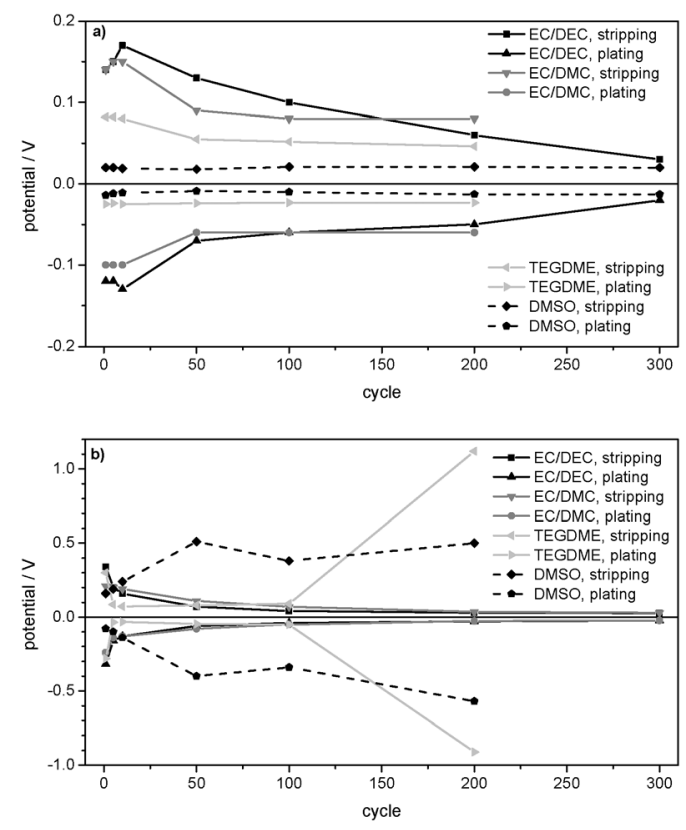

Fig. 9 Maximum overpotentials of subsequent Li plating/stripping processes in $\mathrm{Li} / \mathrm{Li}$ symmetrical cells with $1 \mathrm{M} \mathrm{LiPF}_{6}$ in EC/DEC (3:7) (EC/DEC), $1 \mathrm{MLiPF}_{6}$ in EC/DMC (1:1) (EC/DMC), $1 \mathrm{M} \mathrm{LiPF}_{6}$ in TEGDME (TEGDME), and $1 \mathrm{M}$ LiTFSI in DMSO (DMSO) as electrolytes at (a) $j=0.01 \mathrm{~mA} \mathrm{~cm}^{-2}$ and (b) $j=0.1 \mathrm{~mA} \mathrm{~cm}^{-2}$.
EC/DEC-based electrolyte in Fig. 3a and b. It can be concluded that lithium deposition and dissolution in $1 \mathrm{M}$ LiTFSI in DMSO electrolyte at the lower current density of $j=0.01 \mathrm{~mA} \mathrm{~cm}{ }^{-2}$ proceed with the same characteristics as described for the other electrolytes above.

Apart from the large extent of electrolyte decomposition and SEI film formation in the TEGDME- and DMSO-based electrolytes, the comparison of the results point at a self-enforcing heterogeneity of lithium deposition and dissolution according to the model in Fig. 3a and b in all investigated electrolytes.

The comparison of the maximum overpotentials in Fig. 9a and $b$ shows that the behaviour of $1 \mathrm{M} \mathrm{LiPF}_{6}$ in EC/DEC $(3: 7)$ and $1 \mathrm{M} \mathrm{LiPF}_{6}$ in EC/DMC $(1: 1)$ confirms the general model of an increase of the surface area and thus a general decrease of the overpotentials during cycling, whereas $1 \mathrm{M} \mathrm{LiPF}_{6}$ in TEGDME and $1 \mathrm{M}$ LiTFSI in DMSO deviate at the current density of $j=0.1 \mathrm{~mA} \mathrm{~cm} \mathrm{~cm}^{-2}$. As deduced from the overpotential profiles and confirmed by EIS (Table 2) and SEM investigations, the deviations of the TEGDME- and DMSO-based electrolytes are due to the formation of thick, resistive surface layers stemming from excessive electrolyte decomposition. In $1 \mathrm{M}$ $\mathrm{LiPF}_{6}$ in TEGDME, this effect occurs only after about 150 lithium plating/stripping cycles, whereas it appears during the whole cycling experiment in $1 \mathrm{M}$ LiTFSI in DMSO electrolyte. As displayed in Table 2, the strong decrease of the overpotentials in the EC/DEC-, EC/DMC- and TEGDME-based electrolytes (during the first 150 cycles) is accompanied by a strong decrease of the lithium/electrolyte surface resistance $R_{\text {surface }}$ between $R_{\text {surface }}$ (OCP, 0 days) and $R_{\text {surface }}$ (50 cycles). SEM confirms the conclusion that this decrease is due to the formation of dendrites and thus an increase in the surface area. Concerning the deviations from the general model of the TEGDME- and DMSO-based electrolytes at $j=0.1 \mathrm{~mA} \mathrm{~cm}^{-2}$, Table 2 indicates a dramatic increase of $R_{\text {surface }}$ in $1 \mathrm{M} \mathrm{LiPF}_{6}$ in TEGDME between cycle no. 50 and cycle no. 230 due to the formation of a resistive surface layer. In contrast, a further decrease of $R_{\text {surface }}$ is observed in the EC/DEC- and EC/DMCbased electrolytes between 50 and 370 cycles. In 1 M LiTFSI in DMSO the surface resistance remains comparably constant during the lithium plating/stripping experiment at $j=0.1 \mathrm{~mA} \mathrm{~cm}{ }^{-2}$.

Table 2 displays the constant surface resistances $R_{\text {surface }}$ on/at the pristine $\mathrm{Li}$ electrodes under OCP conditions. Constant values of $R_{\text {surface }}$ were reached after $x=2$ days in the TEGDME-based electrolyte, $x=4$ days in the EC/DMC-based electrolyte, $x=6$ days in the EC/DEC-based electrolyte and $x=7$ days in the DMSO-based electrolyte. The EC/DEC- and EC/DMC-based electrolytes show very high surface resistances, whereas $1 \mathrm{M} \mathrm{LiPF}_{6}$ in TEGDME and $1 \mathrm{M}$ LiTFSI in DMSO present lower values. The order of the surface resistances of the pristine $\mathrm{Li}$ electrode after cell assembly $R_{\text {surface }}$ (OCP, 0 days) as well as after reaching the constant surface resistance $R_{\text {surface }}$ (OCP, $x$ days) corresponds to the following order of the maximum overpotentials in the first cycle at $j=0.01 \mathrm{~mA} \mathrm{~cm}^{-2}$ in Fig. 9a: EC/DEC-, EC/DMC-, TEGDME- and DMSO-based electrolyte. This analogy confirms the deduction that a large SEI resistance on the pristine $\mathrm{Li}$ electrode is directly connected to a high overpotential for lithium deposition and dissolution. 
The strong increase of the overpotential due to severe electrolyte decomposition that was observed in the TEGDME- and DMSO-based electrolytes at $j=0.1 \mathrm{~mA} \mathrm{~cm}^{-2}$ (Fig. 9b) does not appear at a current density of $j=0.01 \mathrm{~mA} \mathrm{~cm}^{-2}$ (Fig. 9a). Hence, the TEGDME- and DMSO-based electrolytes also behave according to the reference model (Fig. 3a and b) at $j=0.01 \mathrm{~mA} \mathrm{~cm}{ }^{-2}$.

The comparison of the maximum overpotentials of the lithium plating/stripping experiments at $j=0.01 \mathrm{~mA} \mathrm{~cm}{ }^{-2}$ (Fig. 9a) and $j=0.1 \mathrm{~mA} \mathrm{~cm} \mathrm{~cm}^{-2}$ (Fig. 9b) confirms the wellknown fact that a higher current density generally induces higher overpotentials. It is also observed that the rate of the overpotential decrease and thus the rate of dendrite formation correlate with the current density.

Besides the general decrease of the electrode surface resistance $R_{\text {surface }}$ during subsequent lithium plating/stripping cycles, EIS investigations (Table 2) indicate an increase of $R_{\text {surface }}$ under OCP conditions. As this increase is supposed to be due to the spontaneous formation of a resistive surface film, the SEI, further investigations were concentrated on the film development and properties.

Therefore, the passivation quality of the SEI and thus the rechargeability of the $\mathrm{Li}$ anode in the particular electrolytes were investigated in $\mathrm{Li}(\mathrm{WE}) / \mathrm{Cu}$ (CE) cells. Fig. 4 shows the potential of the $\mathrm{Li} \mathrm{WE}$ in a $\mathrm{Li} / \mathrm{Cu}$ cell. Besides the already discussed development of the overpotential profiles it can be observed that the duration and thus the capacity of the lithium deposition process on the Li electrode increase during repeated cycling. In these cells the $\mathrm{Cu} \mathrm{CE}$ basically works as a substrate, on which lithium from the WE is deposited and then dissolved again. The overpotential drop at the end of each deposition process indicates that the lithium deposition process on the WE is limited by the amount of deposited lithium on the CE. The difference between the capacities of the deposition and dissolution processes therefore refers to the lithium loss due to electrolyte degradation/lithium corrosion during and after deposition of lithium on the $\mathrm{Cu}$ electrode and due to the loss of electronic contact to dendritic deposited lithium. The increasing capacity of the lithium re-deposition on the WE shows that the lithium loss on the $\mathrm{CE}$ is reduced. This indicates that a passivating and ion conducting film is formed on the

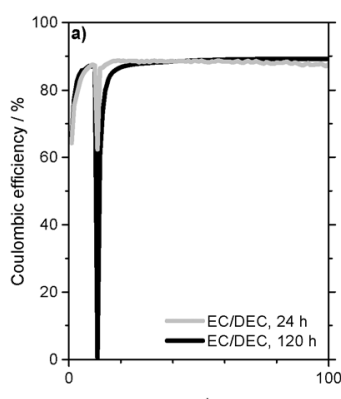

cycle

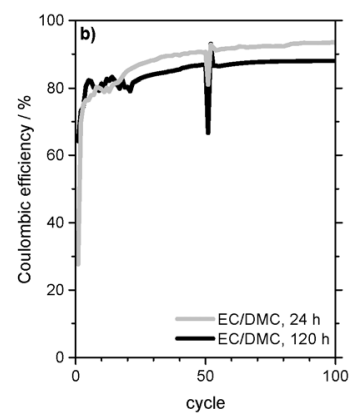

cycle
Fig. 10 Coulombic efficiency of $\mathrm{Li}$ plating/stripping cycles at $j=$ $0.1 \mathrm{~mA} \mathrm{~cm}{ }^{-2}$ in Li (WE)/Cu (CE) cells with (a) $1 \mathrm{M} \mathrm{LiPF}_{6}$ in EC/DEC (3:7) (EC/DEC) and (b) $1 \mathrm{M} \mathrm{LiPF}_{6}$ in EC/DMC (1:1) (EC/DMC) as electrolytes with self-discharge steps at 24 and $120 \mathrm{~h}$ rest time (in the 11th cycle for $\mathrm{EC} / \mathrm{DEC}$, in the 51st cycle for EC/DMC). deposited lithium, which remains mechanically intact on the $\mathrm{Cu}$ surface also when lithium is dissolved again.

For further investigations of the passivation quality of this film, self-discharge experiments have been carried out. In these experiments lithium is deposited on a $\mathrm{Cu} \mathrm{CE}$ and dissolved again after a rest time of $24 \mathrm{~h}$ or $120 \mathrm{~h}$. The comparison of the Coulombic efficiency of this lithium deposition and dissolution cycle with deposition and dissolution cycles without a rest time shows how much active lithium is 'lost' during the rest time. The higher the loss of lithium in the rest time, the lower is the passivation quality of the SEI.

Fig. 10a presents the development of the charge capacity of the WE and the Coulombic efficiency of subsequent lithium deposition/dissolution on the $\mathrm{Cu} \mathrm{CE}$ in $1 \mathrm{M} \mathrm{LiPF}_{6}$ in EC/DEC (3:7) electrolyte. The Coulombic efficiency increases during the first cycles, which indicates the formation of a passivating film on the deposited lithium.

Furthermore, it can be deduced that this film (partly) remains on the $\mathrm{Cu}$ electrode when lithium is dissolved. A rest time of $24 \mathrm{~h}$ in the 11th cycle causes a drop in the Coulombic efficiency from $87 \%$ to $62 \%$ (Table 3 ). The resulting capacity loss due to self-discharge/further lithium corrosion during the rest time is $0.028 \mathrm{~mA} \mathrm{~h}$. As the Coulombic efficiency of the following 12th cycle is again $86 \%$ and thus almost as high as before the rest time, it can be concluded that the passivation quality of the SEI basically remains the same during the rest time.

Also the overpotential profiles of the cycles before and after the rest time (Fig. 11a) indicate that the following 12th cycle only differs in the lithium dissolution process at the WE. This is due to the lower amount of lithium deposited on the WE after the rest time in the 11th cycle.

From Fig. 10a it can also be seen that the Coulombic efficiency drops from $87 \%$ to $0 \%$ between the 10 th cycle and the 11th cycle, which includes 5 days of rest time (Table 3). This indicates a complete corrosion of the deposited lithium on the $\mathrm{Cu} \mathrm{CE}$ during the rest time. The following 12 th cycle shows a Coulombic efficiency of $60 \%$ and it takes another 8 cycles to reach $87 \%$. As the Coulombic efficiencies and thus the overpotential profiles of lithium dissolution (Fig. 11b) after the selfdischarge cycle are also similar to those of the SEI formation in the first cycles of the experiment (compare Fig. 4), it can be deduced that in contrast to the experiment with a rest time of $24 \mathrm{~h}$ the passivating film on the $\mathrm{Cu}$ foil is now lost during the rest time and has to be formed again.

Table 3 Coulombic efficiencies of Li plating/stripping cycles in Li(WE)/ $\mathrm{Cu}(\mathrm{CE})$ cells with $1 \mathrm{M} \mathrm{LiPF}_{6}$ in EC/DEC (3:7), $1 \mathrm{M} \mathrm{LiPF}_{6}$ in EC/DMC (1:1), $1 \mathrm{M}$ $\mathrm{LiPF}_{6}$ in TEGDME and $1 \mathrm{M} \mathrm{LiTFSI}$ in DMSO after 50 and 100 lithium plating/ stripping cycles (cyc.) at $j=0.1 \mathrm{~mA} \mathrm{~cm}^{-2}$ (for EC/DEC and EC/DMC) and $j=0.01 \mathrm{~mA} \mathrm{~cm}^{-2}$ (for TEGDME and DMSO) and after a rest time of $24 \mathrm{~h}$ and $120 \mathrm{~h}$ (rest time/self-discharge test in the 51 st or * 11th cycle)

\begin{tabular}{llllll}
\hline & 50 cyc. & 100 cyc. & 200 cyc. & $24 \mathrm{~h}$ & $120 \mathrm{~h}$ \\
\hline EC/DEC & $88-89 \%$ & $88-89 \%$ & $88 \%$ & $62 \% *(-25 \%)$ & $0 \%^{*}(-87 \%)$ \\
EC/DMC & $87-91 \%$ & $88-93 \%$ & & $81 \%(-10 \%)$ & $67 \%(-26 \%)$ \\
TEGDME & $43-51 \%$ & $43-54 \%$ & $53 \%$ & $12 \%(-30 \%)$ & - \\
DMSO & $20-33 \%$ & $28-63 \%$ & $55-82 \%$ & $0 \%(-33 \%)$ & -
\end{tabular}



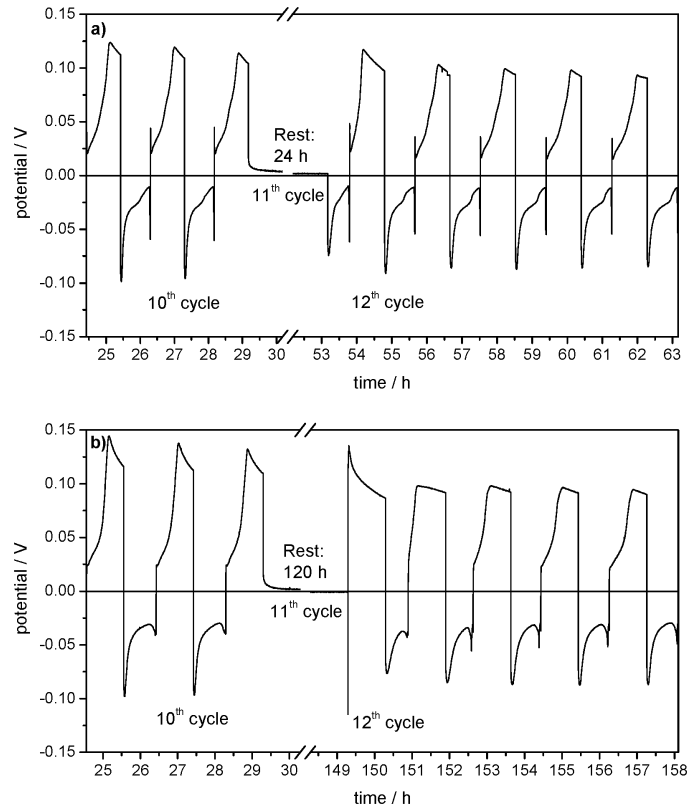

Fig. 11 Overpotential profiles of lithium plating/stripping cycles with a rest time of (a) $24 \mathrm{~h}$ and (b) $120 \mathrm{~h}$ in the 11th cycle in Li (WE)/Cu (CE) cells with $1 \mathrm{M} \mathrm{LiPF}_{6}$ in EC/DEC $(3: 7)$ as the electrolyte at $j=0.1 \mathrm{~mA} \mathrm{~cm}{ }^{-2}$.

Analogous to the experiments in the EC/DEC-based electrolyte, the passivating properties of the surface film formed by the other electrolytes were investigated (Table 3 ). These experiments were carried out at $j=0.1 \mathrm{~mA} \mathrm{~cm}{ }^{-2}$ for $1 \mathrm{M} \mathrm{LiPF}_{6}$ in EC/DMC (1:1) electrolyte (Fig. 10b) and at $j=0.01 \mathrm{~mA} \mathrm{~cm}^{-2}$ for $1 \mathrm{M} \mathrm{LiPF}_{6}$ in TEGDME and $1 \mathrm{M}$ LiTFSI in DMSO electrolytes. Also in these electrolytes it can be observed that the Coulombic efficiencies increase continuously to a certain plateau during the first cycles. It can be concluded that the electrolyte decomposition compounds of these electrolytes also form a passivating film on the $\mathrm{Cu}$ electrode.

In these experiments, the passivation quality of the SEI in different electrolytes differs strongly (Table 3). The lithium plating/stripping experiments in Li/Cu cells with $1 \mathrm{M} \mathrm{LiPF}_{6}$ in EC/DEC (3:7) and $1 \mathrm{M} \mathrm{LiPF}_{6}$ in EC/DMC (1:1) electrolytes show Coulombic efficiencies of $87-95 \%$. Although these values are considerably higher than those observed for the TEGDME- and DMSO-based electrolytes (at $j=0.01 \mathrm{~mA} \mathrm{~cm}^{-2}$ ), they are not sufficient for a reasonable rechargeable operation of the lithium metal anode.

In $1 \mathrm{M}$ LiTFSI in DMSO and $1 \mathrm{M} \mathrm{LiPF}_{6}$ in TEGDME electrolytes the large lithium corrosion prevents the formation of a passivating SEI at $j=0.1 \mathrm{~mA} \mathrm{~cm}{ }^{-2}$. Even at the lower current density of $j=0.01 \mathrm{~mA} \mathrm{~cm}{ }^{-2}$ these electrolytes show lower Coulombic efficiencies than the carbonate-based electrolytes at $j=0.1 \mathrm{~mA} \mathrm{~cm}{ }^{-2}$.

At $j=0.01 \mathrm{~mA} \mathrm{~cm}{ }^{-2}$ the lithium plating/stripping experiments in the TEGDME-based electrolyte show Coulombic efficiencies with high fluctuation and average values of only $43-57 \%$, which indicate a poor passivation quality of the SEI. This is also confirmed by the comparably high capacity loss in the self-discharge experiment.
In the DMSO-based electrolyte Coulombic efficiencies between $55 \%$ and $82 \%$ are observed after $c a .200$ cycles. Self-discharge experiments at various cycles show a complete loss of the deposited lithium by corrosion in only $24 \mathrm{~h}$. Accordingly, the passivation quality of the SEI in the DMSO-based electrolyte is poorer than that of the TEGDME-based electrolyte (compare Table 3). A reasonable operation of both electrolytes in rechargeable batteries with a lithium metal anode is not possible, though the electrolytes have been proposed for lithium/air batteries.

From Table 3 it is also clear that the Coulombic efficiencies of the subsequent lithium deposition and dissolution on the $\mathrm{Cu}$ foil and the Coulombic efficiencies of the self-discharge experiments with rest times of 24 and $120 \mathrm{~h}$ are higher in $1 \mathrm{M} \mathrm{LiPF}_{6}$ in EC/DMC (1:1) electrolyte than in $1 \mathrm{M} \mathrm{LiPF}_{6}$ in EC/DEC $(3: 7)$. In both electrolytes, the SEI cannot prevent continuous lithium corrosion and thus self-discharge occurs during the rest times. A comparison of the Coulombic efficiencies of the selfdischarge experiments for 1 and 5 days in $1 \mathrm{M} \mathrm{LiPF}_{6}$ in the EC/DMC (1:1) electrolyte shows that with a five times longer rest time, the additional capacity loss due to self-discharge is only doubled. The lithium corrosion reaction with the electrolyte is slowed down.

\section{Experimental}

The two carbonate-based standard electrolytes, $1 \mathrm{M} \mathrm{LiPF}_{6}$ in EC/DEC (3:7) and $1 \mathrm{M} \mathrm{LiPF}_{6}$ in EC/DMC (1:1), were used as commercially available (UBE, battery grade $99.9 \%$ ). The $1 \mathrm{M}$ $\mathrm{LiPF}_{6}$ in TEGDME electrolyte was prepared from $\mathrm{LiPF}_{6}$ salt (Sigma-Aldrich, 99.9\%) and TEGDME solvent (Acros Organics, extra pure 99.9\%), and for preparation of 1 M LiTFSI in DMSO electrolyte LiTFSI salt ( $3 \mathrm{M}, 99.9 \%$ ) was dissolved in DMSO (Acros Organics, extra pure 99.9\%). TEGDME and DMSO were dried using molecular sieves and LiTFSI was dried for $72 \mathrm{~h}$ at $110{ }^{\circ} \mathrm{C}$ in a Pfeiffer HiCube vacuum pump with an average vacuum of $10^{-7}$ mbar. LiPF $_{6}$ was used as commercially available (Sigma-Aldrich, 99.9\%).

The constant current cycling experiments and electrochemical impedance spectroscopy were performed in three-electrode Swagelok ${ }^{\circledR}$ cells containing a $\mathrm{Li} \mathrm{WE}$ and a $\mathrm{Li} / \mathrm{Li}^{+} \mathrm{RE}$ in combination with a $\mathrm{Li}$ or $\mathrm{Cu} \mathrm{CE}$. The $\mathrm{Li}$ foil was used untreated as commercially available (Rockwood Lithium, 99.9\%), whereas the $\mathrm{Cu}$ foil was washed with distilled water and ethanol and dried in a Büchi ${ }^{\circledR}$ B585 glass oven $\left(24 \mathrm{~h}\right.$ at $200{ }^{\circ} \mathrm{C}$ and below $3.0 \times 10^{-2} \mathrm{mbar}$ ). For the cells containing $\mathrm{LiPF}_{6}$ salt a stack of three separators was used: a polypropylene/polyethylene/polypropylene (PP/PE/PP) Celgard ${ }^{\circledR} 2325$ separator, a polypropylene (PP) Freudenberg ${ }^{\mathbb{R}} 2190$ separator and again a PP/PE/PP Celgard ${ }^{\mathbb{R}}$ 2325 separator. For the DMSO-based electrolyte, the Freudenberg ${ }^{\mathbb{R}}$ separator was replaced by a glass fiber Whatman ${ }^{\circledR}$ separator (grade GF/D). The separators were dried in a Büchi ${ }^{\mathbb{B}}$ B585 glass oven at $50{ }^{\circ} \mathrm{C}\left(\right.$ Celgard $\left.^{\mathbb{R}}\right), 90{ }^{\circ} \mathrm{C}$ (Freudenberg ${ }^{\mathbb{R}}$ ) and $300{ }^{\circ} \mathrm{C}\left(\right.$ Whatman ${ }^{\mathbb{R}}$ ) in a vacuum below $3.0 \times 10^{-2}$ mbar for 18 hours.

Due to the great sensitivity of lithium metal and other components in the cell to air and moisture, an argon filled 


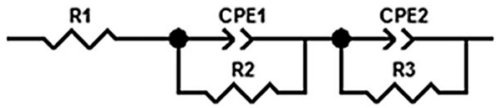

Fig. 12 Equivalent circuit for the interpretation of the electrochemical impedance spectra.

glove box (UNIlab by MBRAUN) with an average $\mathrm{H}_{2} \mathrm{O}$ and $\mathrm{O}_{2}$ content below $0.1 \mathrm{ppm}$ was used for the storage of lithium, mixing and storage of the electrolytes, Swagelok ${ }^{\mathbb{B}}$ cell assembly and sample preparation for the SEM measurements.

The constant current cycling experiments were carried out in a dry oven (BINDER) at $20{ }^{\circ} \mathrm{C}$ using a MACCOR Series 4000 battery test system (MACCOR, INC.). In the experiments with $\mathrm{Li} / \mathrm{Li}$ symmetrical cells a constant current density of $j=0.1 \mathrm{~mA} \mathrm{~cm}^{-2}$ or $j=0.01 \mathrm{~mA} \mathrm{~cm} \mathrm{~cm}^{-2}$ was applied for 1 hour for charging and the same time for discharging. The potential of the $\mathrm{Li} \mathrm{WE}$ was detected against a $\mathrm{Li} / \mathrm{Li}^{+} \mathrm{RE}$. The cut-off potential was set to $+4 \mathrm{~V} v$ s. $\mathrm{Li} / \mathrm{Li}^{+}$for charge (deposition, plating) and $-4 \mathrm{~V} v$ s. $\mathrm{Li} / \mathrm{Li}^{+}$ for discharge (dissolution, stripping).

Also in the experiments in $\mathrm{Li}(\mathrm{WE}) / \mathrm{Cu}(\mathrm{CE})$ cells the potential of the WE was detected against a $\mathrm{Li}^{-} \mathrm{Li}^{+} \mathrm{RE}$. A constant current of $j=0.1 \mathrm{~mA} \mathrm{~cm}{ }^{-2}$ or $j=0.01 \mathrm{~mA} \mathrm{~cm}{ }^{-2}$ was applied for 1 hour for discharging the Li WE. For the dissolution of lithium deposited on the $\mathrm{Cu} \mathrm{CE}$ the same current was applied until lithium was completely dissolved and the potential reached the cut-off condition. In the self-discharge experiments the cell rested in OCP conditions for 1 or 5 days after lithium was deposited on the $\mathrm{Cu} \mathrm{CE}$.

EIS was measured using a Solartron SI 1287 potentiostat in combination with a Solartron SI 1260 impedance/gain phase analyser. The spectra were detected between $1 \mathrm{mHz}$ and $1 \mathrm{MHz}$ with an amplitude of $5 \mathrm{mV}$. They were analysed using ZView Ver. $3.2 \mathrm{~b}$ of Scribner Ass. Inc., and were interpreted by the equivalent circuit in Fig. 12. $R 1$ corresponds to the Ohmic resistance of the electrolyte $R_{\text {electrolyte. }}$ The sum of $R 2$ and $R 3$ refers to the semicircle shown in the Nyquist plots, which was interpreted as the 'Li electrode surface resistance' $\left(R_{\text {surface }}=\right.$ $R 2+R 3)$ of both electrodes.

In order to investigate the surface of the Li electrodes, after the constant current cycling experiments the Swagelok ${ }^{\mathbb{R}}$ cells were disassembled. The sample electrodes were dried under vacuum and transferred to the scanning electron microscope in a sealed vessel. SEM was performed using an Auriga field emission (FE)-SEM Crossbeam Workstation of Carl Zeiss with an acceleration voltage of $3 \mathrm{kV}$.

\section{Conclusions}

The interpretation of the overpotentials of lithium deposition and dissolution on lithium metal allowed an in situ observation of different stages of these processes. By comparative investigations of several liquid electrolytes through constant current cycling experiments combined with EIS and SEM analyses, a detailed model of lithium deposition and dissolution processes has been proposed. In addition to the understanding so far, our model regards that the Li dissolution and deposition processes/mechanisms change during cycling, which can be clearly seen from development of overpotentials during cycling. Furthermore, mutual influences of SEI formation vs. heterogeneous lithium deposition and dissolution, and vice versa were identified.

The evolved method opens a new perspective which allows a direct, fast and non-destructive analysis of the behaviour of the lithium metal anode in liquid electrolytes. It can be transmitted to other electrolytes and for battery systems using alternative metal anodes, such as sodium or magnesium.

Furthermore, the rate of lithium corrosion under open circuit conditions could be investigated.

Generally, it can be stated that charge and discharge of the lithium metal anode in $1 \mathrm{M} \mathrm{LiPF}_{6}$ in EC/DEC (3:7), $1 \mathrm{M} \mathrm{LiPF}_{6}$ in EC/DMC (1:1), $1 \mathrm{M} \mathrm{LiPF}_{6}$ in TEGDME or $1 \mathrm{M}$ LiTFSI in DMSO electrolytes results in dendrite formation and electrolyte decomposition. The electrolytes show the formation of a SEI on lithium metal. By analysis of the Coulombic efficiencies in lithium plating/stripping and self-discharge experiments, it is witnessed that the Li electrode shows poor rechargeability in all investigated electrolytes. Especially the TEGDME- and DMSO-based electrolytes are not suitable for the operation with the rechargeable lithium metal anode at reasonable current densities.

\section{Acknowledgements}

The authors wish to thank the German Ministry of Education and Research (BMBF) for funding of this work through the project 'MEET-HiEnD' (03X4634A).

\section{Notes and references}

1 M. Winter, J. O. Besenhard, M. E. Spahr and P. Novák, Adv. Mater., 1998, 10, 725-763.

2 M. Winter, Z. Phys. Chem., 2009, 223, 1395-1406.

3 J. M. Tarascon and M. Armand, Nature, 2001, 414, 359-367.

4 C. O. Laoire, S. Mukerjee, K. M. Abraham, E. J. Plichta and

M. A. Hendrickson, J. Phys. Chem. C, 2010, 114, 9178-9186.

5 S. A. Freunberger, Y. Chen, Z. Peng, J. M. Griffin, L. J. Hardwick, F. Bardé, P. Novák and P. G. Bruce, J. Am. Chem. Soc., 2011, 133, 8040-8047.

6 R. Wagner, N. Preschitschek, S. Passerini, J. Leker and M. Winter, J. Appl. Electrochem., 2013, 43, 481-496.

7 C. Brissot, M. Rosso, J. N. Chazalviel and S. Lascaud, J. Power Sources, 1999, 81-82, 925-929.

8 J. O. Besenhard, M. W. Wagner, M. Winter, A. D. Jannakoudakis, P. D. Jannakoudakis and E. Theodoridou, J. Power Sources, 1993, 44, 413-420.

9 H. Schranzhofer, J. Bugajski, H. J. Santner, C. Korepp, K. C. Möller, J. O. Besenhard, M. Winter and W. Sitte, J. Power Sources, 2006, 153, 391-395.

10 K. Xu, Chem. Rev., 2004, 104, 4303-4418. 
11 P. Verma, P. Maire and P. Novák, Electrochim. Acta, 2010, 55, 6332-6341.

12 E. Peled, D. Golodnitsky and G. Ardel, J. Electrochem. Soc., 1997, 144, L208-L210.

13 K. Kanamura, H. Tamura, S. Shiraishi and Z.-i. Takehara, J. Electroanal. Chem., 1995, 394, 49-62.

14 E. Peled, J. Electrochem. Soc., 1979, 126, 2047-2051.

15 T. Schedlbauer, U. C. Rodehorst, C. Schreiner, H. J. Gores and M. Winter, Electrochim. Acta, 2013, 107, 26-32.

16 D. Aurbach, E. Zinigrad, Y. Cohen and H. Teller, Solid State Ionics, 2002, 148, 405-416.

17 J. B. Goodenough and Y. Kim, Chem. Mater., 2009, 22, 587-603.

18 Y. S. Cohen, Y. Cohen and D. Aurbach, J. Phys. Chem. B, 2000, 104, 12282-12291.

19 K. Hayashi, Y. Nemoto, S.-i. Tobishima and J.-i. Yamaki, Electrochim. Acta, 1999, 44, 2337-2344.

20 X. Zhang, R. Kostecki, T. J. Richardson, J. K. Pugh and P. N. Ross, J. Electrochem. Soc., 2001, 148, A1341-A1345.

21 M. Egashira, H. Takahashi, S. Okada and J.-i. Yamaki, J. Power Sources, 2001, 92, 267-271.

22 V. S. Bryantsev and M. Blanco, J. Phys. Chem. Lett., 2011, 2, 379-383.

23 V. S. Bryantsev, V. Giordani, W. Walker, M. Blanco, S. Zecevic, K. Sasaki, J. Uddin, D. Addison and G. V. Chase, J. Phys. Chem. A, 2011, 115, 12399-12409.

24 S. E. Sloop, J. K. Pugh, S. Wang, J. Kerr and K. Kinoshita, Electrochem. Solid-State Lett., 2001, 4, A42-A44.

25 X. Sun, H. Lee, X.-Q. Yang and J. McBreen, Electrochem. Solid-State Lett., 2002, 5, A248-A251.
26 B. D. McCloskey, R. Scheffler, A. Speidel, D. S. Bethune, R. M. Shelby and A. C. Luntz, J. Am. Chem. Soc., 2011, 133, 18038-18041.

27 C. Laoire, S. Mukerjee, E. J. Plichta, M. A. Hendrickson and K. Abraham, J. Electrochem. Soc., 2011, 158, A302-A308.

28 H.-G. Jung, J. Hassoun, J.-B. Park, Y.-K. Sun and B. Scrosati, Nat. Chem., 2012, 4, 579-585.

29 D. Xu, Z.-l. Wang, J.-j. Xu, L.-l. Zhang and X.-b. Zhang, Chem. Commun., 2012, 48, 6948-6950.

30 D. Aurbach and E. Granot, Electrochim. Acta, 1997, 42, 697-718.

31 D.-J. Lee, J. Hassoun, S. Panero, Y.-K. Sun and B. Scrosati, Electrochem. Commun., 2012, 14, 43-46.

32 R. Black, S. H. Oh, J.-H. Lee, T. Yim, B. Adams and L. F. Nazar, J. Am. Chem. Soc., 2012, 134, 2902-2905.

33 K. Yoshida, M. Nakamura, Y. Kazue, N. Tachikawa, S. Tsuzuki, S. Seki, K. Dokko and M. Watanabe, J. Am. Chem. Soc., 2011, 133, 13121-13129.

34 S. S. Zhang and T. R. Jow, J. Power Sources, 2002, 109, 458-464. 35 K. Tasaki, A. Goldberg and M. Winter, Electrochim. Acta, 2011, 56, 10424-10435.

36 K. C. Möller, T. Hodal, W. K. Appel, M. Winter and J. O. Besenhard, J. Power Sources, 2001, 97-98, 595-597.

37 S. Jankowsky, M. M. Hiller, R. Stolina and H. D. Wiemhöfer, J. Power Sources, 2015, 273, 574-579.

38 E. Barsoukov and J. R. Macdonald, Impedance Spectroscopy: Theory, Experiment, and Applications, John Wiley \& Sons, 2005.

39 K. Naoi, M. Mori and Y. Shinagawa, J. Electrochem. Soc., 1996, 143, 2517-2522.

40 I. Yoshimatsu, T. Hirai and J. I. Yamaki, J. Electrochem. Soc., 1988, 135, 2422-2427. 